\title{
Insight into the Mechanism of Defensin Neutralization from CryoEM Structural Studies
}

\author{
P.L. Stewart, ${ }^{*}$ J.G. Smith, ${ }^{* *}$ M. Silvestry, ${ }^{*}$ S. Lindert, ${ }^{*}$ W. Lu, ${ }^{* * *}$ and G.R. Nemerow** \\ *Department of Molecular Physiology and Biophysics, Vanderbilt University Medical Center, 2215 \\ Garland Avenue, Nashville, TN 37232 \\ **Department of Immunology and Microbial Science, The Scripps Research Institute, La Jolla, CA \\ 92037 \\ ***Institute of Human Virology, University of Maryland School of Medicine, Baltimore, MD \\ 21201
}

Defensins are peptides of the innate immune system with both antibacterial and antiviral activity. Humans defensins are classified in two groups, $\alpha$ - and $\beta$-defensins. The six human $\alpha$-defensins, HNP1-4, HD5, and HD6, differ in their tissue distribution and expression patterns from the $\beta$ defensins [1]. Both $\alpha$ - and $\beta$-defensins are small peptides with three intramolecular disulfide bonds (Fig. 1). There is strong evidence that a major bactericidal mechanism of defensins is membrane disruption, and lipid bilayer interactions are facilitated by their amphipathicity and net positive charge. Their role in antiviral immunity, particularly for nonenveloped viruses such as human adenovirus (HAdV), is poorly understood. Previous studies have shown that human alpha-defensins inhibit HAdV by preventing virus uncoating and release of the endosomalytic protein VI during cell entry [2]. Therefore, HAdV remains trapped in the endosomal/lysosomal pathway.

We have used a combination of biochemical, structural, molecular genetic, and infection studies to investigate the mechanism of neutralization [3]. The results indicate that defensin binding to HAdV is species specific and that thousands of defensin molecules bind with low micromolar affinity to a sensitive serotype. In contrast, only a low level of binding is observed to a resistant serotype. Also we found that neutralization is dependent upon a correctly folded defensin molecule with the three disulfide bonds intact, suggesting specific molecular interactions between defensin and the virion. We performed a cryoEM analysis of the complex of an Ad vector with a neutralizing defensin, HD5. The Ad vector we chose for these studies is Ad5.F35, which has the HAdV5 capsid pseudotyped with the short HAdV35 fiber. A data set of 3,000 cryoEM particle images was acquired on an FEI Polara microscope (Fig. 2) and was used to generate a three dimensional reconstruction at $12 \AA$ resolution (Fig. 3A). When compared to an Ad5.F35 cryoEM reconstruction without HD5 [4], we observed significant density attributable to HD5 around the Ad fiber in the Ad5.F35+HD5 complex (Fig. 3B). The structure of the Ad5.F35+HD5 complex was further analyzed by docking and subtracting the crystal structure of the penton base with an N-terminal fiber peptide (PDB 1X9T). The difference map suggested a model for the Ad defensin interaction involving a negatively charged and polar region of the fiber that is only found in sensitive serotypes (18-DTET-21 in HAdV2) (Fig. 3C). In contrast, resistant serotypes have a positively charged sequence at this location in fiber. Our model for the Ad defensin interaction is supported by infectivity studies of virus chimeras comprised of capsid components from sensitive and resistant serotypes.

References

[1] R.I. Lehrer, Curr. Opin. Hematol. 14 (2007) 16. 
[2] J.G. Smith and G.R. Nemerow, Cell Host Microbe 3 (2008) 11.

[3] J.G. Smith, M. Silvestry, S. Lindert, W. Lu, G.R. Nemerow, and P.L. Stewart, Submitted.

[4] S.D. Saban et al., J. Virol. 80 (2006) 12049.

[5] This research was supported by NIH grants F32 AI072936 (JGS), T32 GM008320 (MS), EY11431 (GRN), HL054352 (GRN), and AI042929 (PLS).

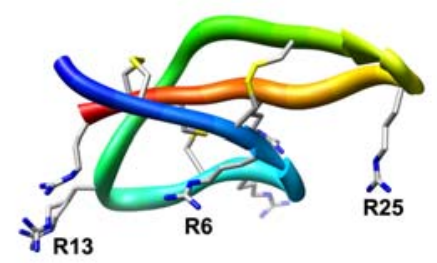

FIG.1. Representation of the HD5 crystal structure (PDB 1ZMP) with the three disulfide bonds and arginine sidechains displayed.

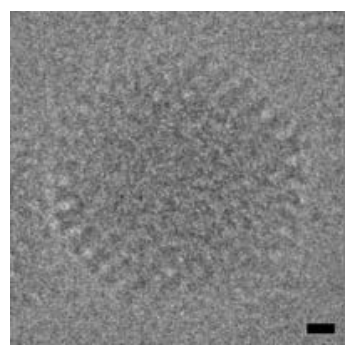

FIG. 2. CryoEM particle image of Ad5.F35+HD5 complex. This particle image was digitally extracted from a cryoelectron micrograph acquired on an FEI Polara (300kV, FEG) microscope with a Gatan UltraScan 4kx4k CCD camera with the sample grid maintained at liquid nitrogen temperature. The micrograph was collected with a magnification of $397,878 \mathrm{X}$ and an underfocus of $2.2 \mu \mathrm{m}$. Scale bar, $100 \AA$.
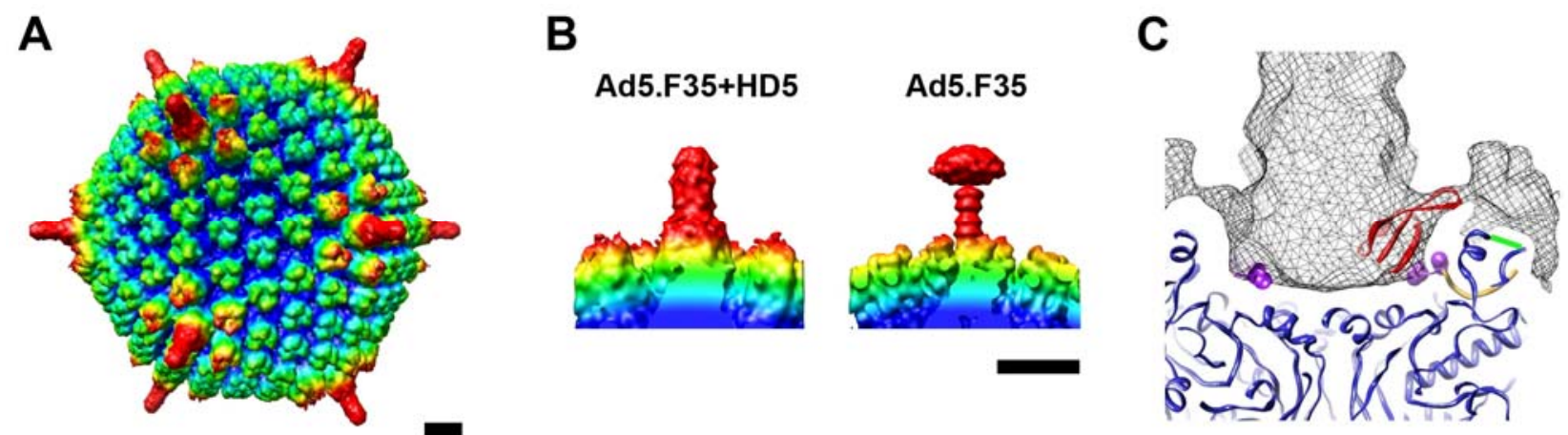

FIG. 3. CryoEM structure of the Ad5.F35+HD5 complex. (A) Structure of Ad5.F35+HD5 at 12A resolution (FSC 0.5 threshold) viewed along an icosahedral 3-fold axis and radially color-coded

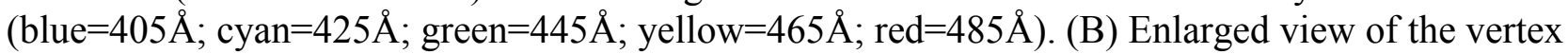
region of Ad5.F35+HD5 compared to Ad5.F35, with both maps filtered to $12 \AA$. (C) A difference map analysis reveals strong density (mesh) near the Ad fiber, which suggested that an HD5 monomer (red) might interact with a negatively charged and polar region of the fiber (magenta spheres). The crystal structure of the HAdV2 penton base (blue) and N-terminal fiber peptide (yellow) (PDB 1X9T) is shown as docked within the Ad5.F35+HD5 structure. The flexible RGD loop of penton base is represented by a green bar. Scale bars, $100 \AA$. 